\title{
Optical properties and fine faulty structure of sapphire crystals grown in low pressure $\mathrm{CO}$ gas atmosphere
}

\author{
V.M.Puzikov, A.Ya.Dan'ko, G.T.Adonkin, N.S.Sidel'nikova, \\ V.F.Tkachenko, A.T.Budnikov \\ S.T.Concern «Institute for Single Crystals», Department of Optical and Constructional Crystals, \\ National Academy of Sciences of Ukraine, 60 Lenin Ave., 61001, Kharkiv, Ukraine
}

\begin{abstract}
Presented in this paper are the results of the study of optical characteristics and fine faulty structure of big sapphire crystals grown in a CO-based low pressure $(0.1 \ldots 0.3$ torr $)$ gas atmosphere. Optimal conditions for annealing optical elements of such crystals have been determined. They provide high transparency of crystals in the UV region and their high resistance to the UV radiation. A perspectivity of using CO medium for growing big sapphire crystals of high optical and structure quality has been confirmed.
\end{abstract}

Keywords: optical transparency, light scattering centers, crystal growth, stoichiometry disordering, evaporation.

Paper received 11.10.99; revised manuscript received 29.11.99; accepted for publication 21.03.00.

\section{Introduction}

Large sapphire crystals with high optical characteristics (free of any light scattering centers) are usually grown in high vacuum $\left(<5 \times 10^{-4}\right.$ torr $)$. The authors have put into practice of growing such crystals a carbon containing $\mathrm{CO}$ based gas medium of a lower vacuum $(\approx 0.1 \ldots 0.3$ torr $)$. This allowed to make the method of horizontally oriented crystallization (HOC) the most efficient one for obtaining large single crystalline sapphire plates (at present to $250 \times 220 \times 30 \mathrm{~mm}^{3}$ ) of high optical quality, any crystallographic orientation on the surface, including (0001) one.

Crystallization of melt in such gas medium has its own peculiarities connected with the exchange processes between melt, gas atmosphere and graphite constructional materials, those in their turn having a strong influence on the important properties of crystals grown under such conditions.

Optical properties and fine faulty structure of sapphire crystals grown in low pressure $\mathrm{CO}$ gas atmosphere were studied in this paper. Crystals were grown from the raw material prepared according to the developed by the authors technique - from cheap alumina used in aluminium industry [2]. This technique provides decrease of concentration of individual impurities down to the level of $5 \cdot 10^{-3}$ mass. $\%$ and lower. Further purification takes place in the process of melt crystallization.

\section{Optical characteristics of crystals}

Investigations, previously carried out by the authors, showed that $\mathrm{Al}_{2} \mathrm{O}_{3}$ melt evaporation in $\mathrm{CO}$-based gas medium at low pressures $(\approx 0.1 \ldots 0.3$ torr $)$ in the case when a partial pressure of $\mathrm{CO}\left(\mathrm{P}_{\mathrm{CO}}\right)$ significantly exceeds that of $\mathrm{CO}_{2}\left(\mathrm{P}_{\mathrm{CO} 2}\right)$ can be even higher under high vacuum conditions $[3,4]$. The necessary ratio of $\mathrm{P}_{\mathrm{CO}}$ and $\mathrm{P}_{\mathrm{CO} 2}$ $\left(\mathrm{P}_{\mathrm{CO}}<<\mathrm{P}_{\mathrm{CO} 2}\right)$ can be achieved by increasing the efficiency of $\mathrm{CO}$ regeneration reaction run on the heated surface of graphite $\left(\mathrm{CO}_{2}+\mathrm{C} \rightarrow 2 \mathrm{CO}\right)$. The high rate of dissociation and evaporation of $\mathrm{Al}_{2} \mathrm{O}_{3}$ in these conditions is connected perhaps with a possibility of its recovery by carbon oxide $[4,5]$.

Active dissociation and evaporation of $\mathrm{Al}_{2} \mathrm{O}_{3}$ from the melt surface during crystallization process probably impede melt stoichiometry disordering. The latter, at $\mathrm{Al}_{2} \mathrm{O}_{3}$ crystallization in Ar atmosphere at a pressure of 760 torr and with reducing constructional materials (graphite, tungsten, molybdenum) used, leads to the formation of light scattering centers $1 \ldots 2 \mathrm{~mm}$ in size and density $10^{6}$ $\mathrm{cm}^{-3}$. This restricts the possibility of using such crystals in optics. It is noteworthy that crystals grown in low pressure $\mathrm{CO}$ atmosphere by the same way as those grown in high vacuum are free of such centers. Certainly, other mechanisms of light scattering centers formation are possible but, as a rule, they are not of a principal character and are easily eliminated. Typical optical characteristics 


\section{V.M. Puzikov et al.: Optical properties and faulty of sapphire crystals ...}

of sapphire crystals grown by the HOC method under different conditions (vacuum $\leq 10^{-4}$ torr, $\mathrm{CO} \approx 0.3$ torr, $\mathrm{Ar}=760$ torr) are presented in Fig. 1 .

In the near UV, visible and IR regions of the spectrum, the crystals grown in high vacuum and low pressure $\mathrm{CO}$ atmosphere practically do not differ, while crystals grown in $\mathrm{Ar}$ (760 torr) have transparency by $0.5 \ldots .2 \%$ worse. In the far region of the spectrum (to $0.2 \mu \mathrm{m}$ ) crystals grown in Ar compare very unfavorably with those grown in vacuum. Some parts of the crystals grown in $\mathrm{CO}$ $(\sim 0.3$ torr $)$ are on the level of grown in vacuum ones. At the same time, the latter are characterized by the lowest resistance to the UV radiation while the highest one is typical for crystals grown in Ar (760 torr), Fig. 2.

As the experiments showed, a characteristic feature of crystals grown in $\mathrm{CO}$ atmosphere ( $\sim 0.3$ torr $)$ is very pronounced nonuniformity of their optical characteristics in the UV region. This refers in a full measure to both: their resistance to the UV radiation and to the transparency in the UV region. These important characteristics of crystals required a more detailed study aiming to find possible ways of improving consumer properties of crystals grown in low pressure $\mathrm{CO}$ atmosphere.

Crystal growth atmosphere is characterized by the value of the redox potential:

$$
\varepsilon=R \cdot T \cdot \ln \left[\left(\frac{P_{A l}^{0}}{P_{A l}}\right)^{2} \cdot\left(\frac{P_{O}}{P_{O}^{0}}\right)^{3}\right]
$$

where $P_{A l}^{0}$ and $P_{O}^{0}$ are the equilibrium partial pressures of $\mathrm{Al}$ and $\mathrm{O}$ above the stoichiometric $\mathrm{Al}_{2} \mathrm{O}_{3}$ melt, $P_{A l}$ and $P_{O}$ are the partial pressures of $\mathrm{Al}$ and $\mathrm{O}$ in the growth medium different from the neutral one with respect to $\mathrm{Al}_{2} \mathrm{O}_{3}$.

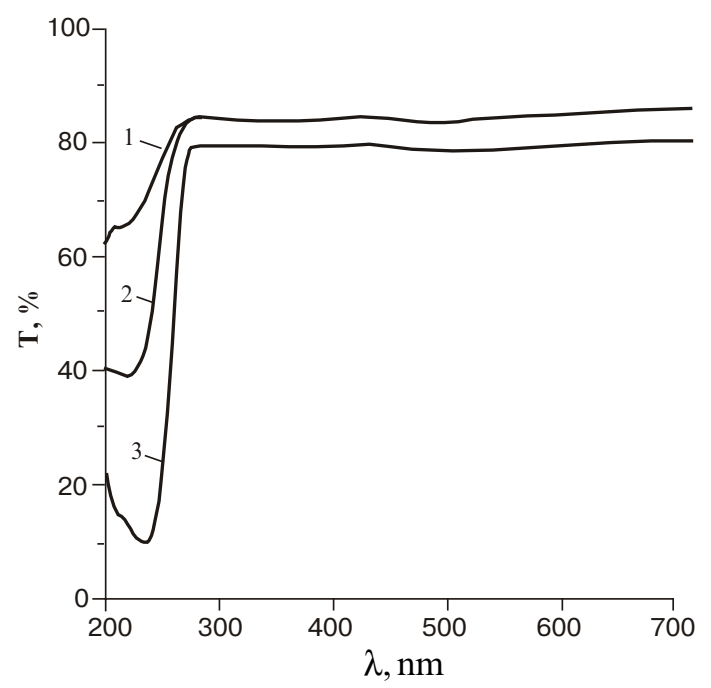

Fig. 1. Transparency of sapphire crystals grown in different atmospheres (thickness of the samples $6 \mathrm{~mm}$ ).
At growing sapphire crystals in the protective gas media, containing the reducing components $\left(\mathrm{C}, \mathrm{CO}, \mathrm{H}_{2}\right)$ which bind free oxygen, the value $e$ is less than zero which results in the stoichiometry disordering by oxygen (formation of $\mathrm{F}^{-}$and $\mathrm{F}^{+}$center). As a rule, $e$ value is impossible to be stabilized under real crystal growth conditions. Therefore, crystals always have certain distribution of anionic vacancy concentration corresponding to particular growth conditions. It is also impossible to provide a uniform distribution of impurities in crystals. Many impurities depending on a charge state (analogously to $\mathrm{F}^{-}$ and $\mathrm{F}^{+}$centers) have absorption bands in the $\mathrm{UV}$ region of the spectrum, thus decreasing transparency of crystals. Proceeding from the existing concepts the resistance to the UV radiation is also connected with both - presence of other valence impurities and intrinsic defects of the lattice.

Optical properties of real crystals grown in the low pressure $\mathrm{CO}$ atmosphere are characterized as follows (Figs 3,4). The transparency in the UV part of the spectrum becomes higher from the beginning to the end and from the bottom of the crucible to the crystal surface. This testifies to a different degree of the stoichiometry disordering and is the result of the change of growth conditions from «more» to «less» reducing during crystallization process and after annealing. The analogous dependence exists also for the resistance to UV radiation. The intensity of the induced coloration becomes higher from the beginning to the end of the crystal. After UV irradiation the initial part of the crystal does not practically change the color but at the end the crystals acquire yellow coloration. As it was noted above the radiation resistance and transparency in the UV region of the spectrum depends on the degree of the stoichiometry disordering and concentration of other valence impurities: $M e^{\mathrm{n}-1}, M e^{\mathrm{n}+1}$ in the crystal.

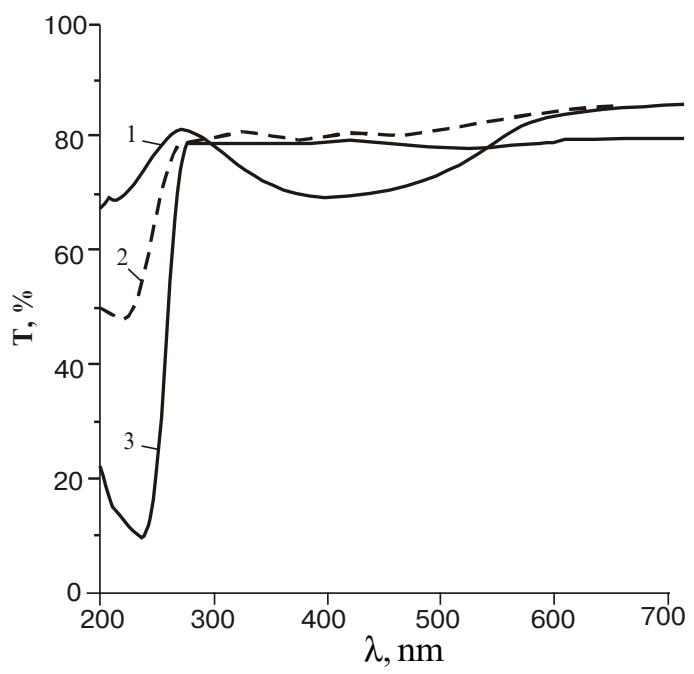

Fig. 2. Transparency of sapphire crystals grown in different atmospheres after UV irradiation (thickness of the samples $6 \mathrm{~mm}$ ) Growth atmosphere: (1) - vacuum $10^{-4}$ torr; (2) - CO, 0.3 torr; (3) Ar, 760 torr. 


\section{V.M. Puzikov et al.: Optical properties and faulty of sapphire crystals ...}

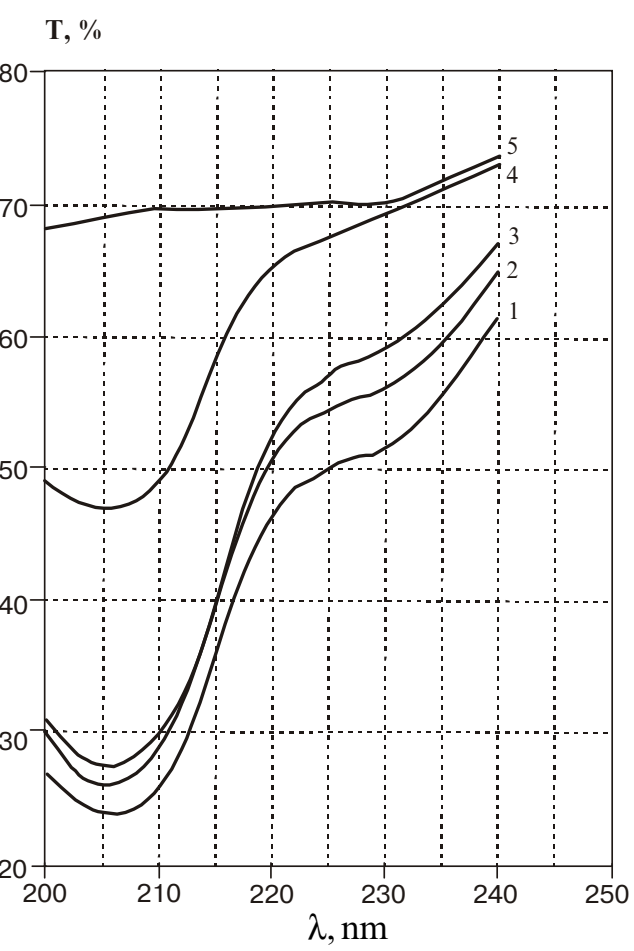

Fig. 3. Transparency of sapphire crystals in the UV region (crystals grown in CO): Sample 1 - the front end of the crystal, ....sample 5 - the end part of the crystal (thickness of the samples $-1 \mathrm{~mm}$ ).

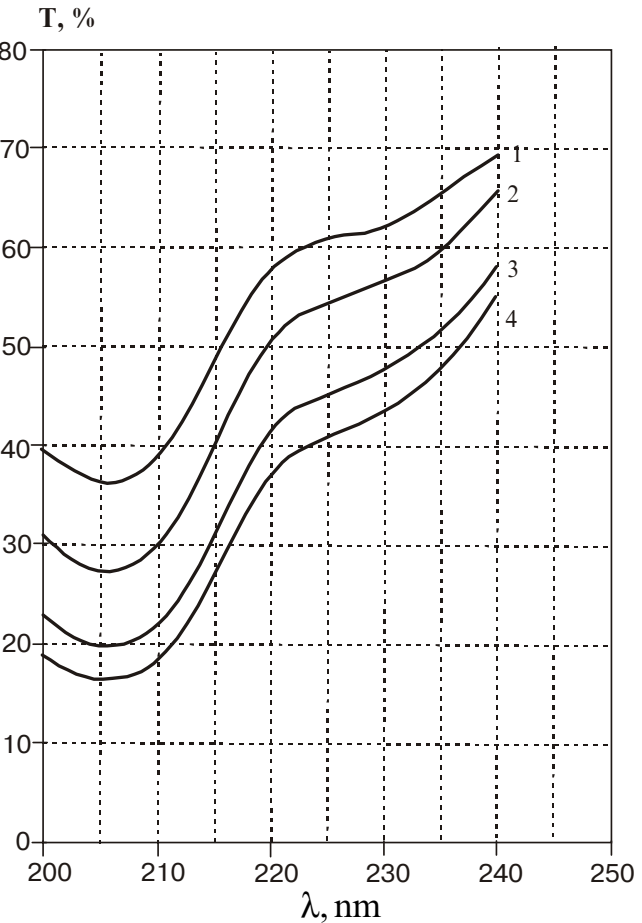

Fig. 4. Transparency of sapphire crystals in the UV region (crystals grown in CO): Sample 1 - the upper part of the crystal, ..., Sample 4 - the lower part of the crystal (thickness of the samples $1 \mathrm{~mm})$.
The results of chemical analysis of the other valence impurities ( $\mathrm{Fe}, \mathrm{Mg}, \mathrm{Ti}, \mathrm{Si}$, ) content at the beginning and at the end of the crystal is presented in Table 1.

Table 1. Content of $\mathrm{Fe}, \mathrm{Mg}$, $\mathrm{Ti}$, and $\mathrm{Si}$ at the beginning and at the end of the crystal

\begin{tabular}{l|c|c|c|c}
\hline \hline \multirow{2}{*}{$\begin{array}{l}\text { Part of the } \\
\text { crystal }\end{array}$} & \multicolumn{4}{|c}{ Mass shares, \% } \\
\cline { 2 - 5 } & $\mathrm{Fe}$ & $\mathrm{Mg}$ & $\mathrm{Ti}$ & $\mathrm{Si}$ \\
\hline Beginning & $5 \cdot 10^{-3}$ & $9 \cdot 10^{-4}$ & $6 \cdot 10^{-4}$ & $2 \cdot 10^{-4}$ \\
\hline End & $3 \cdot 10^{-3}$ & $1.8 \cdot 10^{-3}$ & $1.5 \cdot 10^{-3}$ & $2 \cdot 10^{-3}$ \\
\hline
\end{tabular}

As one can see from the Table, the beginning of the crystal has a higher concentration of $M e^{\mathrm{n}-1}(\mathrm{Fe}, \mathrm{Mg})$ and its end $-M e^{\mathrm{n}+1}(\mathrm{Ti}, \mathrm{Si})$. This being so, the concentration of anionic vacancies having an effect on the charge state of the impurities at the beginning of the crystal is higher as well.

These results allow to assume that in this case the impurities $\mathrm{Me}^{4+}(\mathrm{Ti}, \mathrm{Si})$ are mainly responsible for the formation of color centers, induced by UV radiation:

$O^{2-}+h v \leftrightarrow O^{-}+e$

Formation of strong color centers proceeds according to the scheme:

$\mathrm{O}^{2-}+\mathrm{Me}^{4+}+h v \rightarrow \mathrm{O}^{-}+\mathrm{Me}^{3+}$

The presence of anionic vacancies, compensating the charge of the impurity atom $\mathrm{Me}^{4+}$ :

$\left(V_{O}^{++}+2 e\right)+M e^{4+} \rightarrow\left(V_{O}^{++}+e\right)+M e^{3+}$

impedes formation of strong color centers connected with these impurities. Thus, that part of the crystal, the concentration of anionic vacancies in which is not sufficient to compensate the charge of $M e^{4+1}$, has low radiation resistance.

It can be expected that annealing of this part in the medium with a higher reducing potential $\varepsilon<0$ by favoring a creation of the elevated concentration of anionic vacancies will thus decrease that of the impurities in this charged state. Such annealing is expected to increase not only the radiation resistance but also the optical transparency in the region of $220 \ldots 230 \mathrm{~nm}$ (absorption bands of $\left.\mathrm{Ti}^{4+}, \mathrm{Si}^{4+}\right)$. Indeed, as the experiments showed, the high temperature annealing of the samples with low resistance to the UV radiation in the medium with high reducing potential leads apart from the increase of radiation resistance to the change of transmission in this region of the spectrum, Figs 5, 6 .

The figures show that a lower transparency in the region of $220 \ldots 230 \mathrm{~nm}$ (absorption bands of $\mathrm{Ti}^{4+}, \mathrm{Si}^{4+}$ ) is also observed besides the absorption band at $\sim 400 \mathrm{~nm}$, related to the formation of the induced color centers $\left(\mathrm{O}^{-}\right)$. After annealing in the reducing atmosphere the sample acquires resistance to the UV radiation. This is confirmed by the absence of the absorption band at $\sim 400 \mathrm{~nm}$; transparency in the region of $220 \ldots 230 \mathrm{~nm}$ rises but more in- 


\section{V.M. Puzikov et al.: Optical properties and faulty of sapphire crystals ...}

tensive become the absorption bands at $\sim 500 \mathrm{~nm}\left(\mathrm{Ti}^{3+}\right)$ and $\sim 205 \mathrm{~nm}$ (F-center).

Further investigations showed that the absorption value at $\sim 205 \mathrm{~nm}$ in the annealed samples significantly depends on their impurity composition and, primarily, on the ratio of impurities that are in four- and bivalent state ( $\left.\mathrm{C}_{\mathrm{Me}^{2+}} / \mathrm{C}_{\mathrm{Me}}{ }^{4+}\right)$. Prevailing of the bivalent impurities results in a higher absorption at this wavelength (naturally, provided the medium potential during the preliminary annealing is the same.

Basing on these results a conclusion can be made that by varying the reducing potential of the crystal (optical

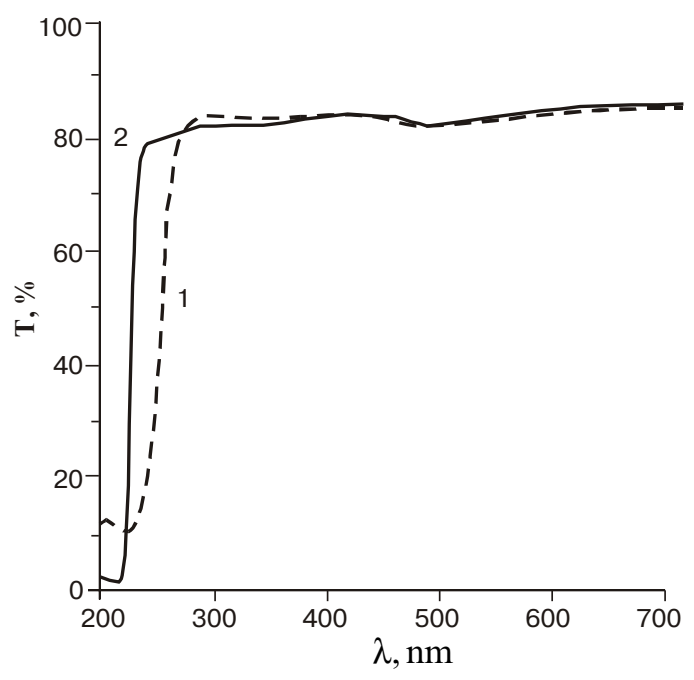

Fig. 5. The effect of annealing $\left(\geq 1900^{\circ} \mathrm{C}\right)$ in the atmosphere with high reducing potential on the properties of crystals in the UV region (the samples $6 \mathrm{~mm}$ in thickness before UV irradiation). (1) - before annealing; (2) - after annealing.

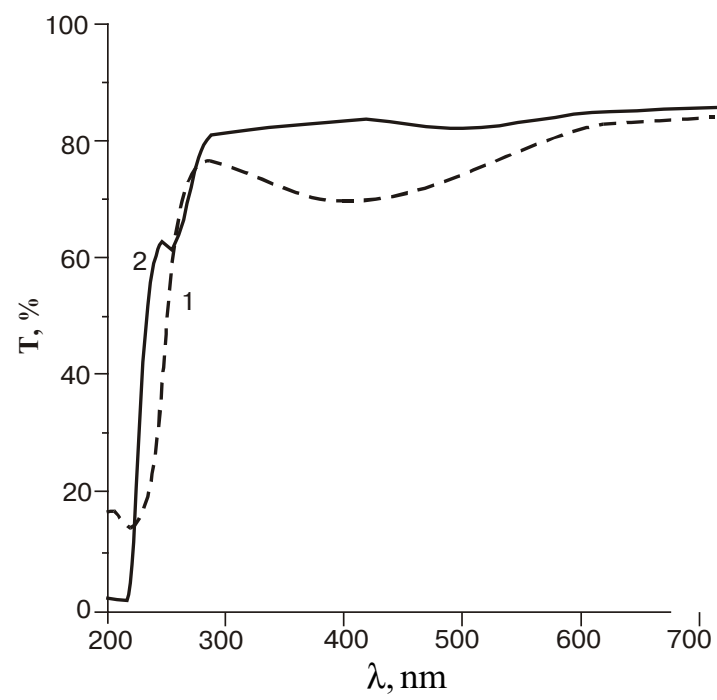

Fig. 6. The effect of annealing $\left(\geq 1900^{\circ} \mathrm{C}\right)$ in the atmosphere with high reducing potential on the properties of crystals in the UV region (the samples $6 \mathrm{~mm}$ in thickness before UV irradiation) (1) - before annealing; (2) - after annealing. element of it) annealing atmosphere one can transform its optical characteristics in the UV region in a rather wide range.

It should be noted that at annealing in high temperature carbon-containing atmosphere the anionic stoichiometry disordering of $\mathrm{Al}_{2} \mathrm{O}_{3}$ is defined by not only a decrease of the partial pressure of oxygen in the vapor phase but also by a possibility of its reduction by the reaction of $\mathrm{Al}_{2} \mathrm{O}_{3}$ with $\mathrm{CO}$. Under standard conditions these reactions are thermodynamically impossible. However, as the calculations showed, they become possible at a compulsory change of the ratio of $\mathrm{CO}$ and reaction products (Fig.7). These conditions can be achieved when conducting this process in vacuum (by lowering the pressure of the reaction products above the surface of $\mathrm{Al}_{2} \mathrm{O}_{3}$ ), by an additional source of $\mathrm{CO}$ used or usage of graphite in the heating zone for $\mathrm{CO}$ regeneration. At the presence of the latter in the heating zone the formed reaction products interact with it:

$\mathrm{C}+\mathrm{CO}_{2}=2 \mathrm{CO}$

$\mathrm{C}+\mathrm{Al}_{2} \mathrm{O}=2 \mathrm{Al}+\mathrm{CO}$

$\mathrm{C}+\mathrm{AlO}=\mathrm{Al}+\mathrm{CO}$

As a result a decrease of partial pressures of the reaction products occurs and an additional source of $\mathrm{CO}$ appears. Therefore, in the $\mathrm{CO}$-based reducing atmosphere the value of $\varepsilon_{O}$ is not ambiguously defined by the equation (1) but depends on the mentioned above parameters defining the reaction rate of $\mathrm{Al}_{2} \mathrm{O}_{3}$ with $\mathrm{CO}$.

The authors found experimentally certain regularities that allow to regulate in a wide range, when necessary, the reducing potential of the annealing medium. This made it possible (even at noticeable concentrations of impurities in crystals $\leq 5 \times 10^{-3}$ mass. $\%$ ) to ensure simultaneously high resistance to the UV radiation and high transparency at the wavelengths up to $205 \mathrm{~nm}$. This result is achieved in two stages (Fig. 8):

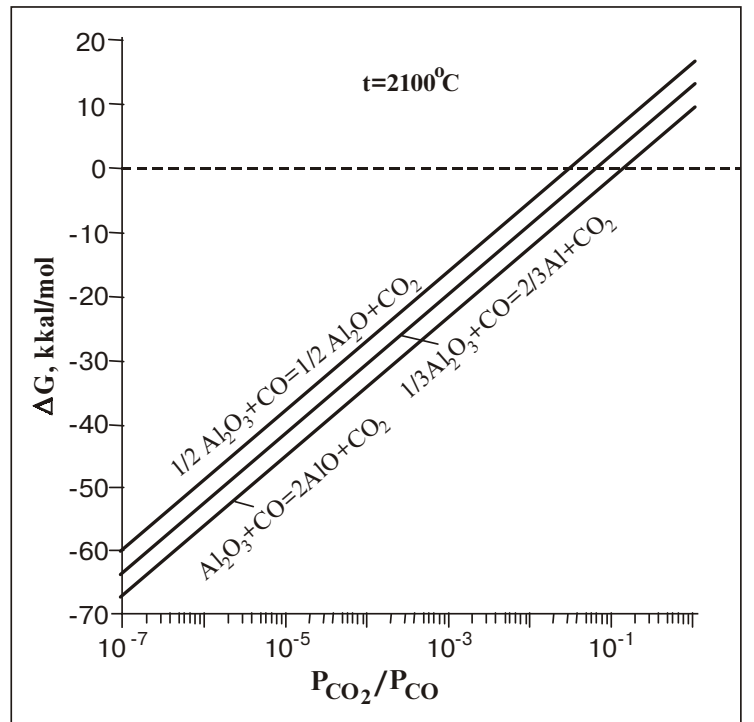

Fig. 7. The calculated dependencies of thermodynamic potential variation on the ratio $P_{\mathrm{CO} 2} / P_{\mathrm{CO}}$ for the reduction of $\mathrm{Al}_{2} \mathrm{O}_{3}$ by $\mathrm{CO}$.

$S Q O, 3(2), 2000$ 


\section{V.M. Puzikov et al.: Optical properties and faulty of sapphire crystals ...}

annealing in the atmosphere with high reducing potential (curve 2) which ensures high resistance to the $\mathrm{UV}$ radiation and transparency up to $220 \mathrm{~nm}$;

annealing in the atmosphere with a potential close to the neutral one (curve 3), which increases transparency at the wavelength $205 \mathrm{~nm}$ (lowering of the F-centers' concentration) while preserving the radiation resistance.

The temperature of annealing is not less than $1900^{\circ} \mathrm{C}$, the duration time is determined experimentally and depends on both - size of the sample and impurity concentration.

\section{Fine faulty structure of crystals}

Structural quality of crystals was studied with the help of a three-crystal X-ray diffractometer (TXD) of high resolution [6]. Angular resolution of the TXD method is about 1 arcsec; the rocking curve halfwidth $\beta \sim 3 \ldots 7$ arcsec, relative variation of crystal lattice parameter $\Delta d / d \sim 1 \cdot 10^{-7}[7]$.

As it is known, such parameters as the form of the diffraction reflection curve (DRC), halfwidth of the rocking curve, integral reflection power $I^{R}$, angular position of DRC are structure sensitive characteristics describing structure quality of the studied crystals. Scanning of the crystal (L-scanning) with respect to the incident X-ray beam allows to receive structure quality characteristics over the cross-section of the sample with the parameters $\beta(L), I^{R}(L), \Delta d / d(L)$ and to characterize the degree of bulk structure quality. Fig. $9(\mathrm{a}, \mathrm{b})$ shows the dependences $\beta(L)$ for the reflection $\{00012\}$ at scanning the sample normally (a) and parallel to the crystal growth direction. (Crystals grown in the $\mathrm{CO}$ gas atmosphere are described in all figures by the curve 2 and those grown in vacuum by the curve 1). Analogous dependences of the integral reflection power $I^{R}(L)$ for the same samples are presented

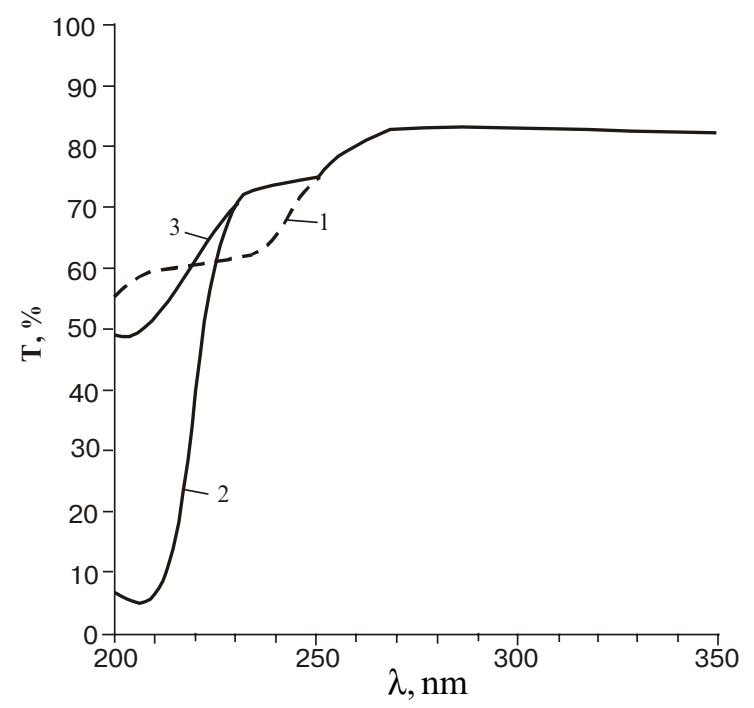

Fig. 8. Transformation of optical characteristics of crystals by a two-stage annealing in the media with different reducing potential $\varepsilon$ (thickness of the samples $2 \mathrm{~mm}$ ).

(1) - before annealing; (2) - after annealing with $\varepsilon<<0$; (3) after a repeat annealing with $\varepsilon<<0$. in Fig. 10 (a, b). As one can see from these figures, there is no significant difference in structure quality charac-

$\beta$, arcsec

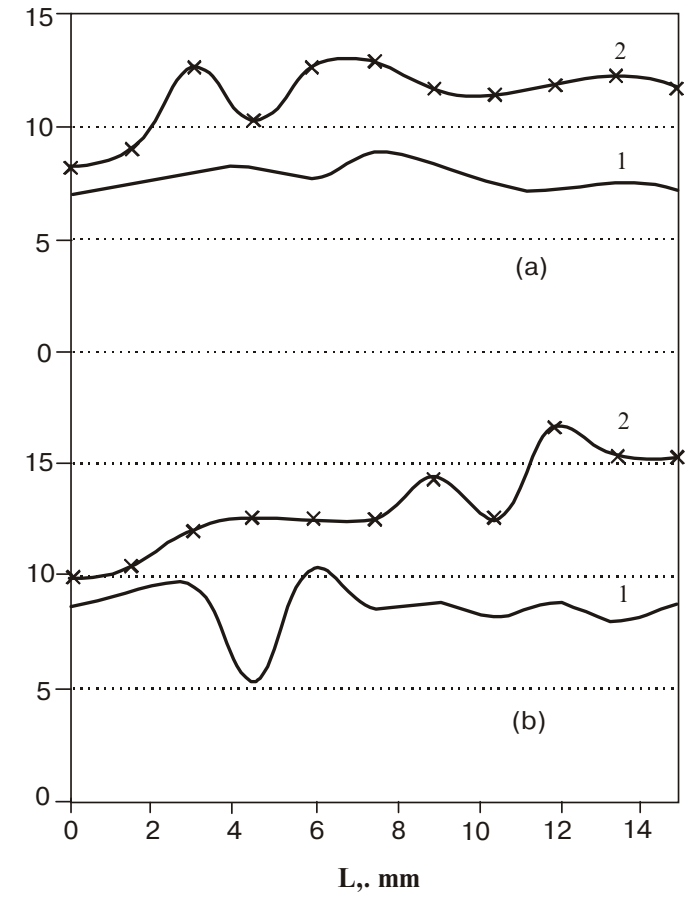

Fig. 9. Behavior of the rocking curve halfwidth $\beta(L)$.

(1) - crystal grown in vacuum; (2) - crystal grown in CO. a) - scanning normally to the growth direction; b) - scanning parallel to the growth direction.

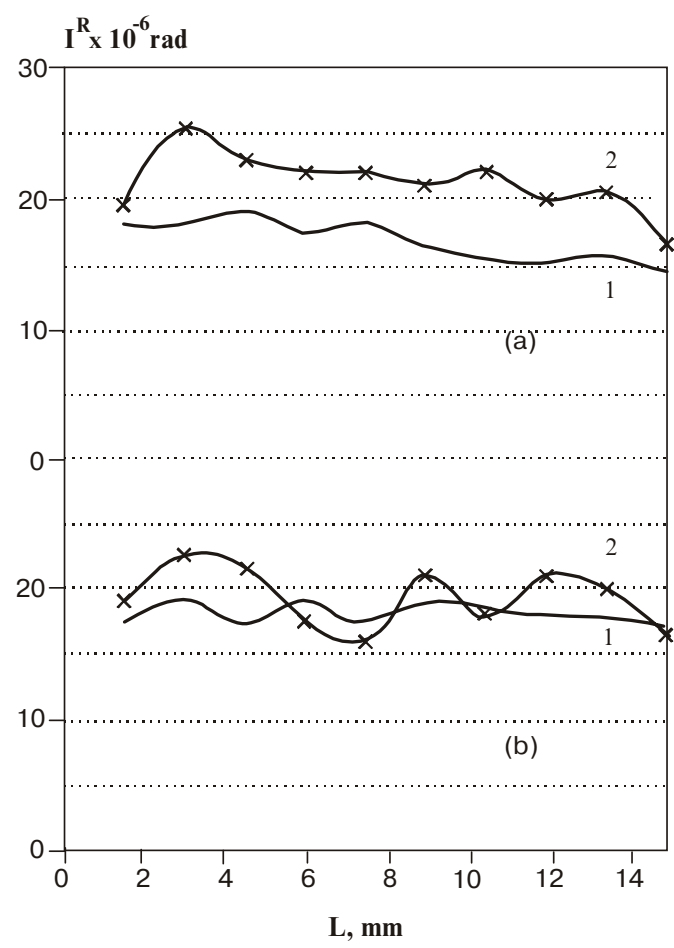

Fig. 10. Behavior of the integral reflection power $I^{R}(L)$.

(1) - crystal grown in vacuum; (2) - crystal grown in CO.

a) - scanning normally to the growth direction; b) - scanning parallel to the growth direction. 


\section{V.M. Puzikov et al.: Optical properties and faulty of sapphire crystals ...}

teristics of crystals grown in $\mathrm{CO}(\sim 0.3$ torr $)$ and in vacuum. No big blocks are observed in all the samples, only low angle boundaries of microblocks with disorientation angles to 15 arcsec can be found.

An insignificant increase of the crystal lattice parameter $C$ at $\Delta C=9.96 \cdot 10^{-4} \mathrm{~A}$ for crystals grown in the $\mathrm{CO}$ atmosphere as compared to those grown in vacuum was noticed. A rather high concentration of anionic vacancies in these crystals and the character of their distribution can probably explain this fact.

\section{Conclusions}

Thus, growth of sapphire crystals in a low pressure ( $\sim 0.1 \ldots 0.3$ torr) CO-based atmosphere ensures their high optical and structure quality, crystals comparing favorably with those grown in high vacuum. With allowance for higher economic properties and possibility to grow crystals by the HOC method bigger than those grown in high vacuum makes this atmosphere more promising and technologic for a mass sapphire production.

\section{References}

1. A.Ya.Dan'ko, N.P.Katrich, Yu.P.Miroshnikov, V.E.Kachala, G.T.Adonkin. A method for growing aluminium oxide crystals. Patent of Ukraine N18923A, 1996 (in Russian).

2. A.Ya.Dan'ko, V.N.Kanischev, N.S.Sidel'nikova, G.T.Adonkin, S.V.Nizhankovskii. A furnace for the preparation of raw material used in crystal growth from melt. Decision on issue of the Patent of 31.08 .99 (in Russian).

3. A.Ya.Dan'ko, G.T.Adonkin, N.S.Sidel'nikova, A.T.Budnikov. Peculiarities of $\mathrm{Al}_{2} \mathrm{O}_{3}$ evaporation in gas atmospheres of Ar, $\mathrm{N}_{2}$ and $\mathrm{CO}$ at low pressures // Functional materials, 1, N2, 1994 (in Russian).

4. A.Ya.Dan'ko, N.S.Sidel'nikova, G.T.Adonkin, A.T.Budnikov.Thermochemical etching and polishing sapphire surface in a gas atmosphere // Functional materials, 4, N1, 1997 (in Russian).

5. A.Ya.Dan'ko, N.S.Sidel'nikova, V.N.Kanischev, G.T.Adonkin, S.V.Nizhankovskii. Purification of fine dispersion $\mathrm{Al}_{2} \mathrm{O}_{3}$ powders in CO-based gas atmosphere // Functional materials, 5, N2, 1998 (in Russian).

6. V.F.Tkachenko, M.A.Rom, A.A.Bibichenko, V.I.Kuznetsov. Three-crystal X-ray diffractometer for the study of crystalline materials // Pribory i tekhnika experimenta, N2, 1992 (in Russian).

7. V.F.Tkachenko, M.A.Rom. Author's Certificate N.1702265. A method of precision measurements of crystal lattice parameters, Bulletine N48, 1991 (in Russian). 